\title{
WTAP is a prognostic marker of high-grade serous ovarian cancer and regulates the progression of ovarian cancer cells
}

This article was published in the following Dove Press journal: OncoTargets and Therapy

\author{
Hai-Lan $\mathrm{Yu}^{1,2}$ \\ Xu-Dong $\mathrm{Ma}^{\mathrm{l}, 2}$ \\ Jin-Fei Tong ${ }^{1,2}$ \\ Jian-Qiong $\mathrm{Li}^{1,2}$ \\ Xiao-Jing Guan ${ }^{1,2}$ \\ Jian-Hua Yang ${ }^{1,2}$ \\ 'Assisted Reproduction Unit, \\ Department of Obstetrics and \\ Gynecology, Sir Run Run Shaw Hospital, \\ Zhejiang University School of Medicine, \\ Hangzhou, People's Republic of China; \\ ${ }^{2}$ Key Laboratory of Reproductive \\ Dysfunction Management of Zhejiang \\ Province, Hangzhou, People's Republic of \\ China
}

Correspondence: Jian-Hua Yang Assisted Reproduction Unit, Department of Obstetrics and Gynecology, Sir Run Run Shaw Hospital, Zhejiang University School of Medicine, Key Laboratory of Reproductive Dysfunction Management of Zhejiang Province, No 3 East Qing Chun Road, Hangzhou 310016, People's

Republic of China

Tel +86I 3989816498

Email yjh2006@zju.edu.cn
Background: The Wilms' tumor suppressor WT1 is reported to work in a range of physiological processes at both transcriptional and posttranscriptional level. WT1associating protein (WTAP), a nuclear protein co-localized with splicing factors, also plays a vital role in cellular function and cancer progression. However, little is known about the role of WTAP in ovarian cancer and the underlying mechanism.

Materials and methods: To evaluate the expression of WTAP, multiple means were applied in clinical tissues, including immunohistochemistry, quantitative reverse transcriptase PCR (qRT-PCR), and Western blot. Two representative ovarian cancer cell lines (3AO and SKOV3) were used to assess the malignant influence of WTAP on proliferation, apoptosis, and migration. To explore its function, WTAP was additionally down-regulated by lentivirus. Results: High expression of WTAP in high-grade serous ovarian carcinoma (HGSOC) predicted a shorter overall survival $(P<0.01)$. Furthermore, WTAP expression was higher in HGSOC, compared with that in normal ovary group $(P<0.01)$, benign ovarian tumor group $(P<0.01)$, and non-HGSOC group $(P<0.05)$. In HGSOC, high expression of WTAP was significantly related with the lymph node metastasis $(P<0.05)$. In ovarian cancer cell lines, cell proliferation and migration were considerably reduced after WTAP was down-regulated, while apoptotic rate was increased. Moreover, the effect of WTAP in 3AO and SKOV3 might be relevant with MAPK and AKT signaling pathways

Conclusion: WTAP is highly expressed in HGSOC, and indicates a worse survival outcome. Therefore, it is highly possible that WTAP has a prognostic implication in the patients of HGSOC. In addition, WTAP down-regulation also plays a tumor suppressor role in $3 \mathrm{AO}$ and SKOV3 cell lines.

Keywords: WTAP, ovarian cancer, diagnosis, tumor suppression

\section{Introduction}

Ovarian cancer is currently the fourth most common cause regarding cancer deaths among females. In 2018, the estimated number of new cases that would be diagnosed as ovarian cancer is about 22,240, and would result in approximately 14,070 deaths in the United States. Epithelial ovarian cancer accounts for more than $90 \%$ of all cases of ovarian cancer, and consists of mucinous, endometrioid, clear cell, high-grade and low-grade serous ovarian cancer, and a few other histological types. The five-year survival of serous ovarian carcinoma (SOC) is $43 \%$, which is lower than other types. ${ }^{1}$ The benign and borderline ovarian tumors always progress slowly and have a much better outcome, ${ }^{2}$ while the malignant ovarian cancer is 
more aggressive, characterized by its rapid metastasis and the occurrence of severe complications. ${ }^{3}$ Unfortunately, due to the absence of obvious symptoms and early screening tests, a majority of epithelial ovarian cancer patients are diagnosed at advanced stages, and $75 \%$ of these patients would finally die of their diseases. ${ }^{4}$ Hence, the importance of researching the early diagnostic method and new prognostic biomarkers is further highlighting.

Wilms' tumor 1-associating protein (WTAP) is a ubiquitously expressed nuclear protein, which was first identified for its specific interaction with WT1 (Wilms' tumor 1), a protein playing an essential role in normal development of the urogenital system. ${ }^{5}$ The function of WT1 is controversial because it mutates in a subset of patients with Wilms' tumor and is always regarded as a cancer suppressor, ${ }^{6}$ but some recent studies have shown that nuclear WT1 expression level was associated with metastatic ovarian cancer. ${ }^{7,8}$ WTAP was also shown to act as an oncogene and was related to malignant tumors closely. For instance, WTAP promoted tumor growth in colorectal cancer through Wnt signaling pathway by negatively regulating WT1. ${ }^{9}$ Furthermore, nuclear WTAP expression was believed to be an independent prognostic biomarker of pancreatic ductal adenocarcinoma. ${ }^{10}$ In renal cell carcinoma, WTAP promoted cancer proliferation via enhancing the stability of CDK2 mRNA by directly binding to CDK2 transcript. ${ }^{11}$ In diffuse large B-cell lymphoma, WTAP not only increased proliferation, but also counteracted Etoposide-mediated apoptosis, which indicated a poor chemotherapy sensitivity. ${ }^{12}$ Noticeably, WTAP gene is located on chromosome $6 \mathrm{q} 25.3$ in human, a region concerned to be associated with ovarian cancer. $^{13}$

To the best of our knowledge, this study is the first investigation to show the WTAP expression in different ovarian tumors, and its relevance to the clinical features of high-grade serous ovarian carcinoma (HGSOC) patients. Besides, the study revealed the influence of WTAP on the malignant biological behaviors of ovarian cancer cell lines.

\section{Materials and methods}

\section{Patients and tissue samples}

The collection and use of human ovary tissues were approved by the Human Research Ethical Committee of the Sir Run Run Shaw Hospital, Zhejiang University School of Medicine. Tissues used for experiment were obtained from patients who underwent ovarian resection at the Sir Run Run Shaw Hospital from June 2015 to November 2017. All recipients and one family member of each donor signed informed consent forms. The tissues were collected and immediately transported in an ice box to the laboratory. Half part of the tissue was frozen in liquid nitrogen for protein and RNA extraction, and the other part was fixed in formaldehyde solution for Immunohistochemistry (IHC). Cases included 17 benign epithelial ovarian tumors, 19 borderline epithelial ovarian tumors, 30 HGSOCs, 17 other epithelial ovarian carcinomas (4 mucinous carcinomas, 2 endometrioid tumors, 6 clear-cell tumors, 1 low-grade SOC, and 4 other types), and 11 normal ovaries undergoing whole uterus and double attachment resection owing to uterine fibroids or adenomyosis.

\section{Immunohistochemistry}

Dehydrate human ovarian tissues were sliced into $4 \mu \mathrm{M}$ sections. A rabbit anti-human WTAP monoclonal antibody (Abcam, ab195380, Shanghai, China) was used to perform IHC staining. The slides were deparaffinized with xylene and rehydrated with alcohol. Citrate buffer was used as antigen retrieval by incubating the slides at $100^{\circ} \mathrm{C}$ for 10 mins. Then, hydrogen peroxide was used to block endogenous peroxidase. Later, tissues were incubated at diluted primary antibody $(1: 100)$ at $4^{\circ} \mathrm{C}$ overnight. HRP-labeled secondary antibody was applied to incubate slides, and DAB substrate solution was used to chromogenesis. Finally, counterstain slides with Hematoxylin. Positive (sections of liver cancer) and negative (sections stained with PBS instead of primary antibody) controls were included in each batch. The percentage of pigmented cells in the visual field was as follows: $<10$ (negative); 10-30 (weak expression); 31-70 (moderate expression); $>70$ (strong expression). Cell stain images were evaluated by an expert pathologist who was blinded as to the method of sampling, and a duplicate scoring was performed to control for errors.

\section{Cell culture and reagents}

The EOC cell line $3 \mathrm{AO}$ was obtained from Cell Biology of Chinese Academy of Sciences (Shanghai, China), SKOV3 cell line was provided by Dr. YD Shen. The application of cell lines was approved by the Human Research Ethical Committee of the Sir Run Run Shaw Hospital, Zhejiang University School of Medicine. The cells were cultured in DMEM/F12 (Gibco, Carlsbad, CA, USA) supplemented with 10\% FBS (Cellmax life, CA, USA). Cell plates were maintained in a $5 \% \mathrm{CO}_{2}$ humidified atmosphere at $37^{\circ} \mathrm{C}$. 


\section{Western blotting}

Proteins were isolated from the snap-frozen ovary tissues and cultured cell lines, extracted in RIPA solution (Beijing Solarbio Technology Co. Ltd., Beijing, China) with a protease inhibitor cocktail (Roche, Shanghai, China). Using a BCA Protein Assay Kit (Thermo Fisher Scientific, Shanghai, China) to quantify the protein expression level. Then, $20 \mu \mathrm{g}$ of each protein sample was separated via 10 $12 \%$ SDS-PAGE and electro-transferred onto polyvinylidene fluoride membranes. Following blockade with PBST containing 5\% nonfat dry milk. Membranes were incubated with primary antibodies against the following molecules overnight at $4{ }^{\circ} \mathrm{C}$ : WTAP (Abcam, ab195380), Cleaved caspase-3 (CST, 9661, Boston, MA, USA), Caspase-3 (CST, 9665), Bcl-2 (CST, 3498), Bax (CST, 2772), E-cadherin (CST, 3195), Vimentin (Abcam, ab925471), p-AKT (Ser473) (CST, 4060), AKT (CST, 4691), p-ERK (CST, 4370), ERK (CST, 4695), p-p38 (CST, 9215), p38 (CST, 8690), $p$-JNK (CST, 4668), JNK (CST, 9258), GAPDH (Abcam, ab9485). Using HRP-conjugated IgG as secondary antibodies, and incubated at $37^{\circ} \mathrm{C}$ for $1 \mathrm{hr}$. Antigen and antibody complexes were detected following an ECL protocol (Millipore, Darmstadt, Germany). Immunoblots were quantified using Image Lab software (Quantity One software, Bio-Rad, CA, USA).

\section{RNA isolation and expression analysis}

Total RNA from snap-frozen ovary tissues and cultured cell lines was isolated using TRIzol reagent (Life Technologies, Carlsbad, CA, USA) according to the manufacturer's protocol, and then underwent reverse transcription to generate complementary DNA using a PrimeScript ${ }^{\mathrm{TM}}$ RT reagent kit (Takara Biotechnology Co. Ltd., Dalian, China). Quantitative real-time PCR was performed using SYBR Green mix (Takara Biotechnology Co. Ltd., Dalian, China) in a Real-Time PCR system (Life Technologies, Carlsbad, CA, USA). Target gene expression was normalized to GAPDH expression, and the relative mRNA abundance was calculated utilizing the $\Delta \Delta \mathrm{Ct}$ method. Real-time PCR was performed using the following specific primers: WTAP (forward) 5'-TTGTAATGCGACTAGCAACCAA-3' and (reverse) 5'- GCTGGGTCTACCATTGTTGATCT-3'.

\section{Lentivirus construction and cell transfection}

$3 \mathrm{AO}$ and SKOV3 cells were transfected with WTAP knockdown lentivirus and negative control vectors, following the manufacturer's instructions (GENECHEM, Shanghai, China). The efficiency of knockdown was examined by quantitative reverse transcriptase PCR (qRT-PCR) and Western blot.

\section{Cell proliferation assays}

For cell proliferation assays, CCK-8 kit (Dojindo, Kumamoto, Japan) was used according to the manufacturer's instruction. Cells were seeded in 96-well plates at a density of $2 \times 10^{3}$ cells per well. After $24,48,72,96,120$, and 144 hrs of culture, $10 \mu \mathrm{L}$ Cell Counting Kit- 8 for each well was added to assess the cell proliferation level after incubation at $37^{\circ} \mathrm{C}$ for another $2 \mathrm{hrs}$, and then read at 450 nm with a microplate reader. All experiments were performed in triplicate.

\section{Colony formation assay}

For the colony formation assay, $2 \times 10^{3}$ cells were plated into six-well plate, and cultured in complete culture medium. After 2 weeks, colonies were fixed with 4\% paraformaldehyde and stained with $0.1 \%$ crystal violet (Beyotime, Beijing, China). Finally, visible colonies were photographed (Nikon, Tokyo, Japan) and counted. All experiments were performed in triplicate.

\section{Apoptosis assay}

For Apoptosis assay, $3 \mathrm{AO}$ and SKOV3 cells were infected with control or WTAP knockdown lentivirus and then cultured for 48 hrs. $2 \times 10^{5}$ cells were harvested and then suspended with binding buffer. Cells were stained with Annexin V-PE and 7-AAD (Becton Dickinson, Oxford, UK) to detect apoptosis using a FACS Calibur flow cytometer. The quantitative analysis of the percentage of positive cells was obtained from using Cell Quest Modfit software. All experiments were performed in triplicate.

\section{Scratch wound-healing assay}

Cells were seeded into six-well plate at the proper density ensuring the $95-100 \%$ monolayer cell confluence in the next day. Then, cells were wounded by dragging a $200 \mu \mathrm{L}$ pipette tip. The primary wound images were acquired, and the healed wound images were taken again 48 hrs later. All experiments were performed in triplicate.

\section{Transwell migration assay}

During this assay, cells suspended with serum-free medium were seeded on the upper layer of a cell culture chamber (Corning, MA, USA) with permeable membrane 
and medium with $10 \%$ FBS was placed below the cell permeable membrane. Following $24 \mathrm{hrs}$ incubation period, the cells that had migrated through the membrane were stained by $0.1 \%$ crystal violet, and then photographed and counted. All experiments were performed in triplicate.

\section{Survival analysis}

GEPIA (http://gepia.cancer-pku.cn/detail.php) is an interactive web server for analyzing the RNA sequencing expression data. ${ }^{14} \mathrm{~A}$ total of 424 HGSOC samples contained in GEPIA were used to analyze expression levels of key genes. ${ }^{15}$ We can customize the high- and lowexpression threshold of the target molecule, and in this study, the median expression of WTAP in all cases is used as the demarcation point to define high and low expression. $P<0.05$ was considered statistically significant.

\section{Statistical analysis}

Dates were presented as the means \pm SEM of at least three independent experiments. All data were analyzed with SPSS version 20 software (IBM, Chicago, IL, USA). All variables were normally distributed, confirming by the Kolmogorov-Smirnov test followed by Q-Q plots analysis. Student's $t$-test was used for comparison between two groups; Fisher's exact test was used for comparison between classification variables; one-way ANOVA was used to compare among more than two groups. The curve of overall survival was analyzed by Kaplan-Meier and $\log$-rank tests. $P<0.05$ was considered statistically significant.

\section{Results}

\section{High expression of WTAP is associated with a poor prognosis in HGSOC}

According to microarray data deposited in GEPIA (http:// gepia.cancer-pku.cn/), a publicly available database, WTAP expression was closely connected with prognosis of HGSOC. Patients with low WTAP expression $(n=212)$ had obviously better overall survival than those with high WTAP expression $(\mathrm{n}=212) \quad(P<0.01$; Figure 1A). Low WTAP expression might be associated with better disease-free survival compared to high WTAP expression, whereas it made no statistical difference $(P=0.078$; Figure $1 \mathrm{~B})$.

\section{Expression of WTAP in different ovarian tissues}

WTAP expression was assessed by IHC on patients' specimens, and the results are represented in Table 1. Among 30 HGSOC tissues, 25 (83.3\%) of them expressed WTAP, and the ratio was greater than those in normal ovary group $(3 / 11,27.3 \% ; P<0.01)$, benign ovarian tumor group $(7 / 17,41.2 \% ; P<0.01)$, and nonHGSOC group $(9 / 17,52.9 \% ; P<0.05)$. Representative IHC staining images were shown in Figure 2A. WTAP was expressed extremely higher in HGSOC tissues than in normal ovaries, according to the Western blot

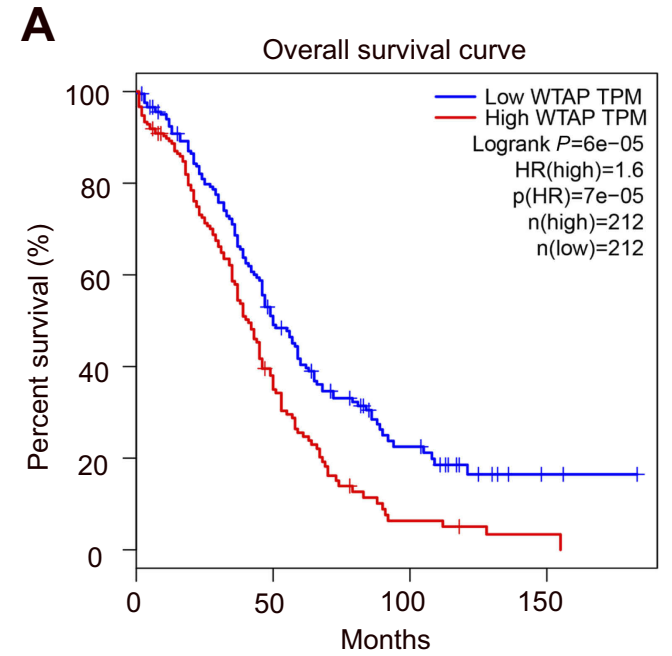

B

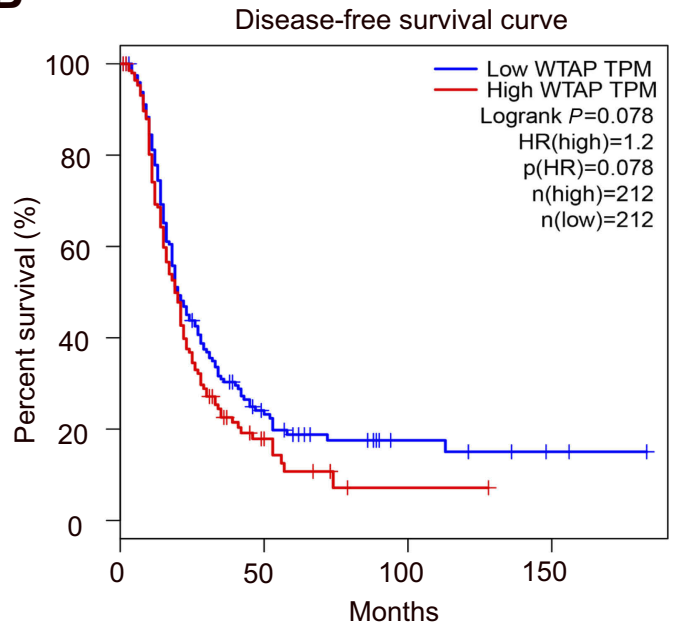

Figure I Overall survival and disease-free survival curves of HGSOC patients based on WTAP expression levels. (A) The overall survival curve of patients with high WTAP expression (red line) and with low WTAP expression (blue line). (B) The disease-free survival curve of patients with high WTAP expression (red line) and with low WTAP expression (blue line).

Abbreviations: HGSOC, high-grade serous ovarian carcinoma; HR, hazards ratio; TPM, transcripts per million. 
Table I WTAP expression in different ovarian tissues

\begin{tabular}{|l|l|l|}
\hline \multirow{2}{*}{ Category } & WTAP staining & Positive rate (\%) \\
\cline { 2 - 3 } & Positive/total & $27.3^{* *}$ \\
\hline Normal ovary & $3 / 11$ & $41.2^{* *}$ \\
Benign ovarian tumor & $7 / 17$ & 63.2 \\
Borderline ovarian tumor & $12 / 19$ & $52.9^{*}$ \\
Non-HGSOC & $9 / 17$ & 83.3 \\
HGSOC & $25 / 30$ & \\
\hline
\end{tabular}

Notes: $* P<0.05$, $* * P<0.01$, vs HGSOC group.

Abbreviation: HGSOC, high-grade serous ovarian carcinoma.

A

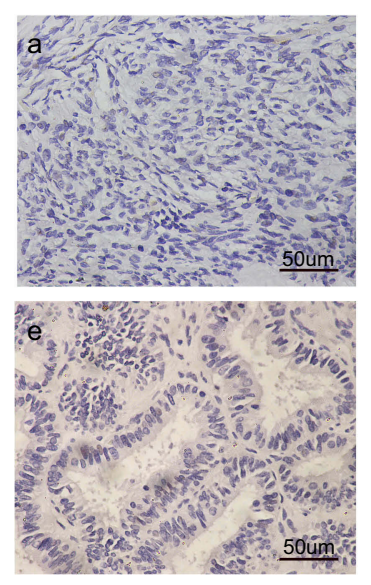

B

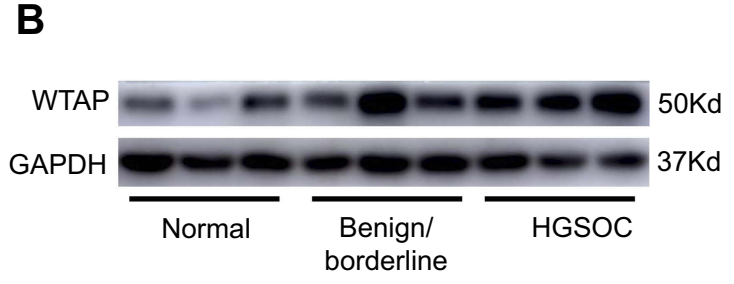

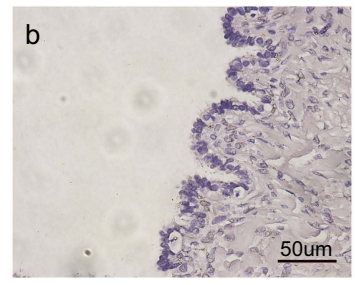

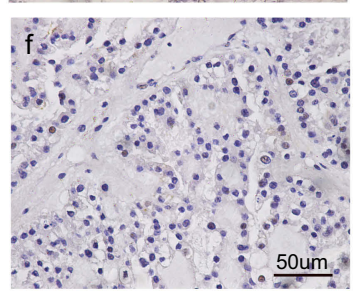

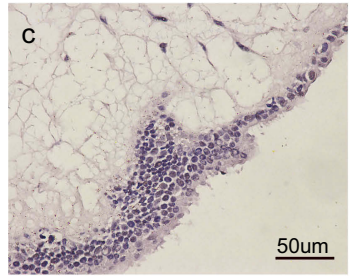

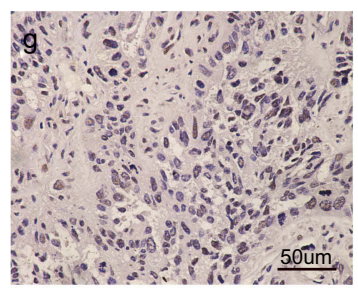

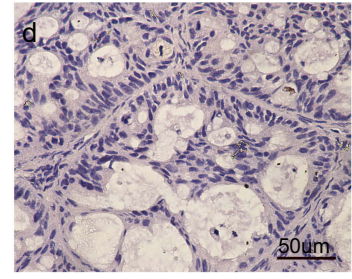

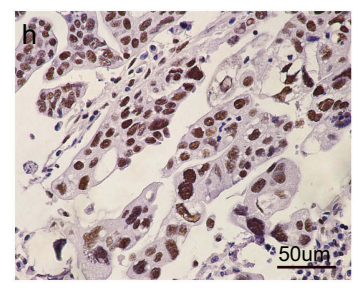

C

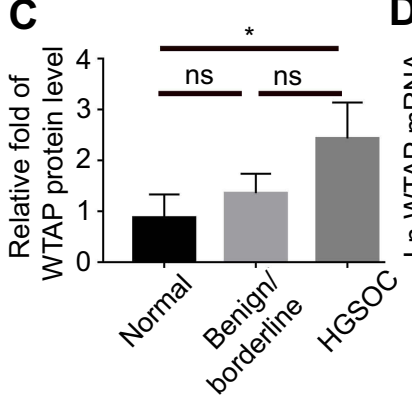

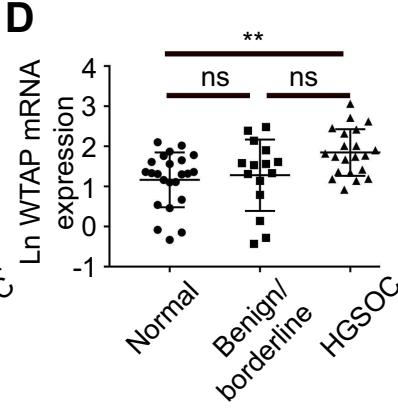

Figure 2 Immunohistochemistry, Western blot and qRT-PCR results of WTAP expression in different ovarian tissues. (A) Representative WTAP immunohistochemistry staining in different types of ovarian tissues ( $\times 400)$ : (a) normal ovary; (b) serous cystadenoma; (c) borderline serous cystadenoma; (d) mucinous cystadenocarcinoma; (e) endometrioid carcinoma; ( $f$ ) clear-cell carcinoma; (g) low-grade serous ovarian cancer; (h) high-grade serous ovarian cancer. (B, C) Protein expression of WTAP in ovarian tissues. (D) Relative mRNA expression of WTAP in ovarian tissues. $* P<0.05, * * P<0.01$.

Abbreviation: ns, non significant.

(Figure $2 \mathrm{~B}$ and $\mathrm{C}, P<0.05$ ) and qRT-PCR (Figure 2D, $P<0.01)$ results. It is obvious that WTAP was abundant in HGSOC tissues, but its function was still elusive.

\section{Relation between WTAP expression and clinicopathological factors in HGSOC patients}

To find out the potential connection between WTAP expression and clinicopathological features in HGSOC patients,
IHC was performed to define the low and high WTAP expression level. Thirty cases of HGSOC with basic and clinicopathological information were collected and divided into two groups. Low-expression group $(\mathrm{n}=10)$ included specimens with negative $(<10 \%)$ and weak $(10-30 \%)$ WTAP expression, while high-expression group $(n=20)$ included specimens with moderate (30-70\%) and strong $(>70 \%)$ WTAP expression. Clinicopathological features were compared between these two groups (Table 2), demonstrating that WTAP expression was higher in cases 
Table 2 Relationship of WTAP expression and clinicopathologic characteristics of HGSOC patients

\begin{tabular}{|c|c|c|c|c|}
\hline \multirow[t]{2}{*}{ Variable } & \multirow[t]{2}{*}{ Total } & \multicolumn{2}{|c|}{ WTAP expression } & \multirow[b]{2}{*}{$P$-value } \\
\hline & & Low $(n=10)$ & High $(n=20)$ & \\
\hline Age (years) & & & & 0.431 \\
\hline$<60$ & 21 & $6(7)$ & $15(14)$ & \\
\hline$\geq 60$ & 9 & $4(3)$ & $5(6)$ & \\
\hline BMI & & & & 0.689 \\
\hline$<24$ & 22 & II (I5.2) & II (I8.8) & \\
\hline$\geq 24$ & 8 & $5(4.3)$ & $3(3.7)$ & \\
\hline Tumor size $(\mathrm{cm})$ & & & & 0.675 \\
\hline$<6$ & 7 & $3(3.7)$ & $4(3.3)$ & \\
\hline$\geq 6$ & 23 & $13(12.3)$ & $10(10.7)$ & \\
\hline Stage & & & & $0.07 I$ \\
\hline $\mathrm{I} / \mathrm{II}$ & 8 & $6(3.7)$ & $2(4.3)$ & \\
\hline $\mathrm{III} / \mathrm{IV}$ & 22 & $8(10.3)$ & $14(\mid \mathrm{I} .7)$ & \\
\hline Grade of differentiation & & & & 0.675 \\
\hline GI & 23 & $13(12.3)$ & $10(10.7)$ & \\
\hline G2/G3 & 7 & $3(3.7)$ & $4(3.3)$ & \\
\hline Lymph node metastasis & & & & $0.013^{*}$ \\
\hline NO & 15 & $\mathrm{II}(7.5)$ & $4(7.5)$ & \\
\hline YES & 15 & $4(7.5)$ & II (7.5) & \\
\hline Volume of ascites $(\mathrm{mL})$ & & & & 0.260 \\
\hline$<500$ & 20 & $9(10.7)$ & II (9.3) & \\
\hline$\geq 500$ & 10 & $7(5.3)$ & $3(4.7)$ & \\
\hline CAI25 (U/mL) & & & & 0.257 \\
\hline$<500$ & 11 & $4(5.9)$ & $7(5.1)$ & \\
\hline$\geq 500$ & 19 & $12(10.1)$ & $7(8.9)$ & \\
\hline
\end{tabular}

Notes: $* P<0.05$. $P$-values were two-tailed and based on the Fisher's exact test; Values in brackets represent the expected count.

Abbreviation: HGSOC, high-grade serous ovarian carcinoma.

with lymph node metastasis $(P<0.05)$. However, WTAP expression was independent of other parameters, such as age, BMI, tumor stage, tumor size, differentiation grade, ascites volume, and CA125 level.

\section{WTAP knockdown influences $3 A O$ and SKOV3 progression in vitro}

$3 \mathrm{AO}$ and SKOV3 were widely used in ovarian cancer cell lines. To further explore the function of WTAP, WTAP knockdown was successfully achieved in $3 \mathrm{AO}$ and SKOV3 cells by lentivirus infection, and its efficiency was validated by Western blot (Figure 3A) and qRT-PCR (Figure 3B).

The influence of WTAP knockdown on cell proliferation was examined by using CCK-8 proliferation assay. After WTAP knockdown, cell proliferation in both $3 \mathrm{AO}$ and
SKOV3 cell lines was impeded (Figure 3C). Furthermore, the WTAP down-regulation reduced growth ability owing to less colonies formed after 14 days than the control group in both cell lines (Figure 3D). The role of WTAP during the process of cell apoptosis was checked by flow cytometry. Higher apoptotic rates were revealed in $3 \mathrm{AO}$ and SKOV3 cells low-expressing WTAP (Figure 3E). Higher cleaved caspase-3 level was also observed, with no change in the ratio of Bax/Bcl-2 (Figure 3F). Taken together, these results suggested that down-regulation of WTAP inhibited $3 \mathrm{AO}$ and SKOV3 proliferation and simultaneously promoted cell apoptosis.

\section{WTAP knockdown suppresses SKOV3}

\section{migration}

According to the transwell (Figure 4A and B) and woundhealing assays (Figure 4C), the down-regulation of WTAP 
A

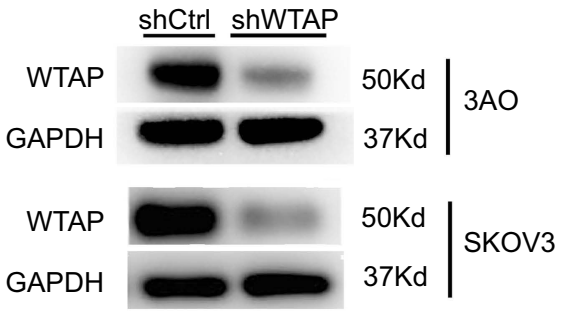

C
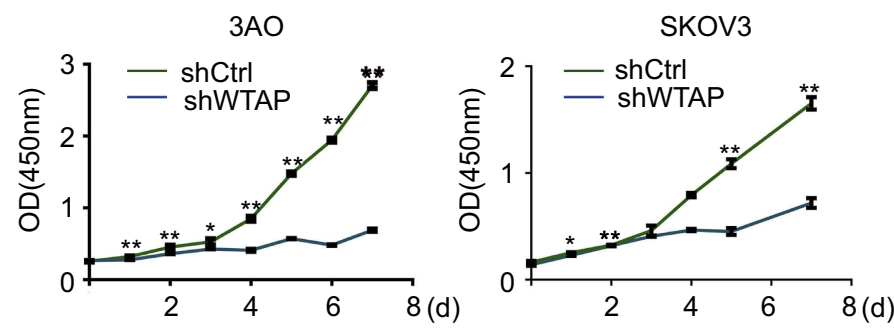

E
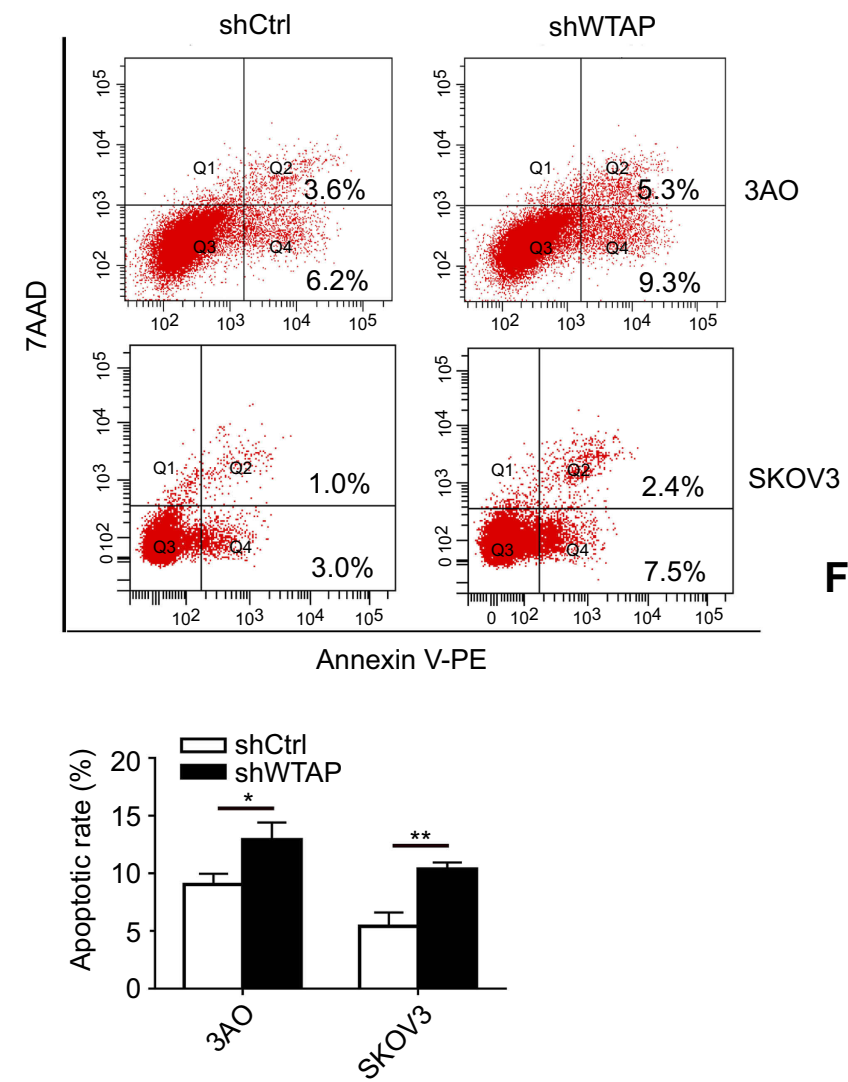

F
B

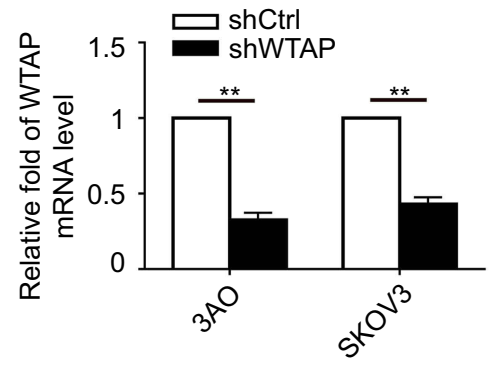

D
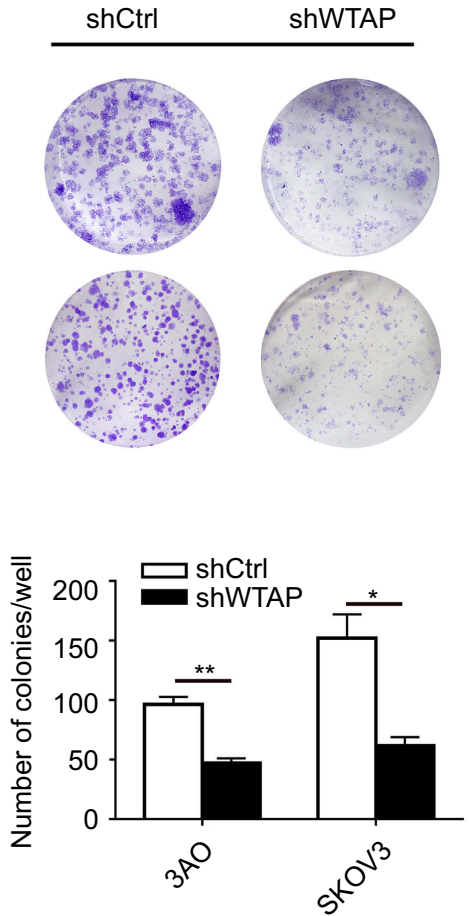

Cleaved caspase-3
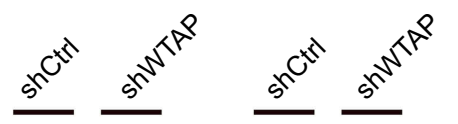

Caspase-3

$19 \mathrm{kd}$

Caspase-3

$\mathrm{Bcl}-2$

GAPDH
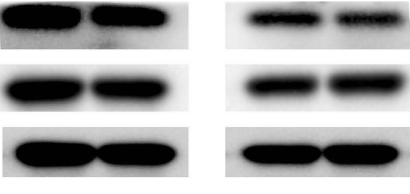

$3 A O$

SKOV3

Figure 3 WTAP regulates cellular proliferation and apoptosis. (A) Protein level of WTAP knockdown efficiency in 3AO and SKOV3 cell lines was detected by Western blot. (B) mRNA level of WTAP knockdown efficiency was detected by qRT-PCR. (C) The influences of WTAP knockdown on cell proliferation were confirmed using the CCK-8 assay. (D) The representative picture of colony formation assay, and the quantification of colonies per well. (E) The representative results of the impact of WTAP knockdown on cell apoptosis, and the quantification of apoptotic rates. (F) The changes of apoptosis-related proteins after down-regulation of $W T A P$. $* P<0.05$, $* * P<0.0$ I. Abbreviation: OD, optical density. 
A

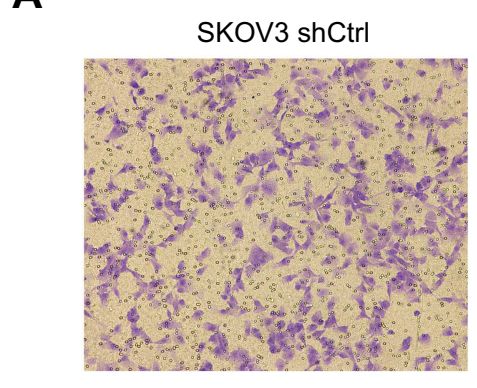

C

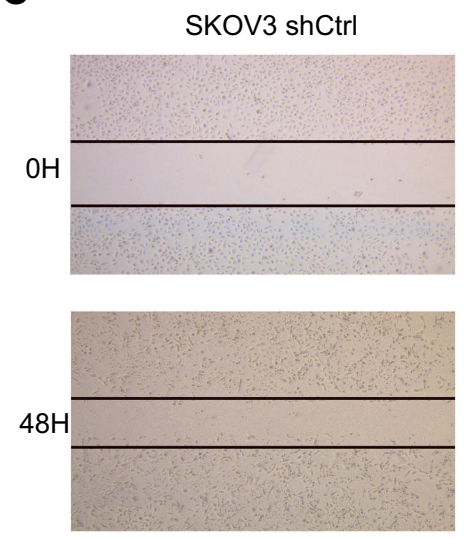

B
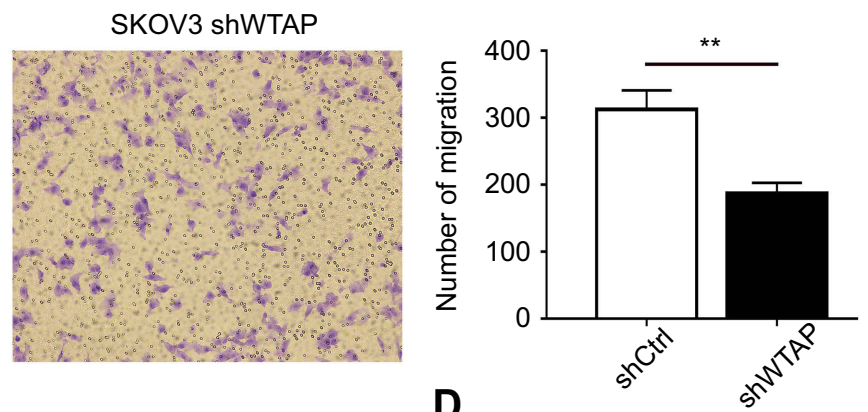

D

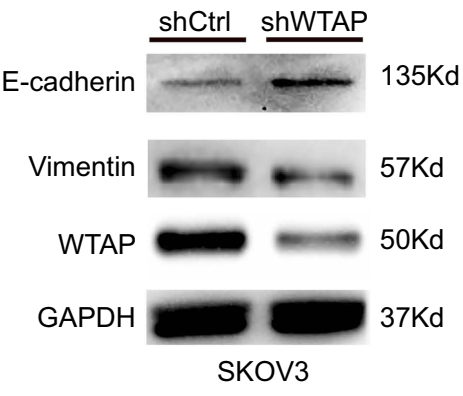

Figure 4 WTAP regulates cellular migration in SKOV3 cell line. (A, C) The effect of WTAP knockdown on SKOV3 migration was detected by transwell assay and scratch wound-healing assay. (B) The results of the transwell assay were quantified. (D) The expression changes of EMT related biomarkers after down-regulation of WTAP. $* * P<0.01$.

Abbreviation: EMT, epithelial-mesenchymal transition.

in SKOV3 cell line-inhibited cell migration, compared to the control group. Relevant biomarkers of epithelialmesenchymal transition (EMT) pathway were further detected, and we found that the down-regulated WTAP increased E-cadherin expression and decreased Vimentin expression (Figure 4D).

\section{Possible signaling pathways contributing to the function of WTAP in $3 A O$ and SKOV3}

After knockdown of WTAP in $3 \mathrm{AO}$ and SKOV3 cell lines, the expression of $p$-AKT, AKT, $p$-ERK, ERK, $p$-JNK, JNK, p38, p-p38 was detected by Western blot. In this study, the promotions of $p$-AKT/AKT, $p$-ERK/ERK, $p$-JNK/JNK, $p$ - $\mathrm{p} 38 / \mathrm{p} 38$ were all decreased after WTAP down-regulation (Figure 5). Thus, it could be speculated that the function of WTAP in $3 \mathrm{AO}$ and SKOV3 cell lines was possibly associated with MAPK and AKT pathways.

\section{Discussion}

Ovarian cancer ranked fourth in cancer deaths among women and had a higher risk of incidence and mortality than other cancers of the female reproductive system. ${ }^{16}$ Despite obvious advances previously in detecting methods and therapies, there is still no effective screening test that can detect ovarian cancer at an early stage, leading to a diagnostic delay.

WTAP was shown to interact with WT1 specifically in vivo and in vitro. The WTAP gene was mapped to human chromosome region identified to associate with ovarian cancer. ${ }^{13}$ As an important member of m6A methyltransferase complex, WTAP has gained recognition in regulating various cellular processes physiologically and pathologically, and was considered as a housekeeping protein for its ubiquitous expression. ${ }^{5}$ The complex catalyzing m6A modification consists of METTL3, METTL14, WTAP, RBM15, VIRMA, HAKAI, and ZC3H13. ${ }^{17}$ It is reported that WTAP alone does not possess any methyltransferase activity, however, it guides the whole complex into nuclear speckles. ${ }^{18}$ RNA-seq analysis revealed that with the depletion of both WTAP and KIAA1429, the nuclear/ cytoplasmic ratio of $73 \%$ mRNAs was increased, indicating that WTAP was involved in mRNA export. ${ }^{19}$ 


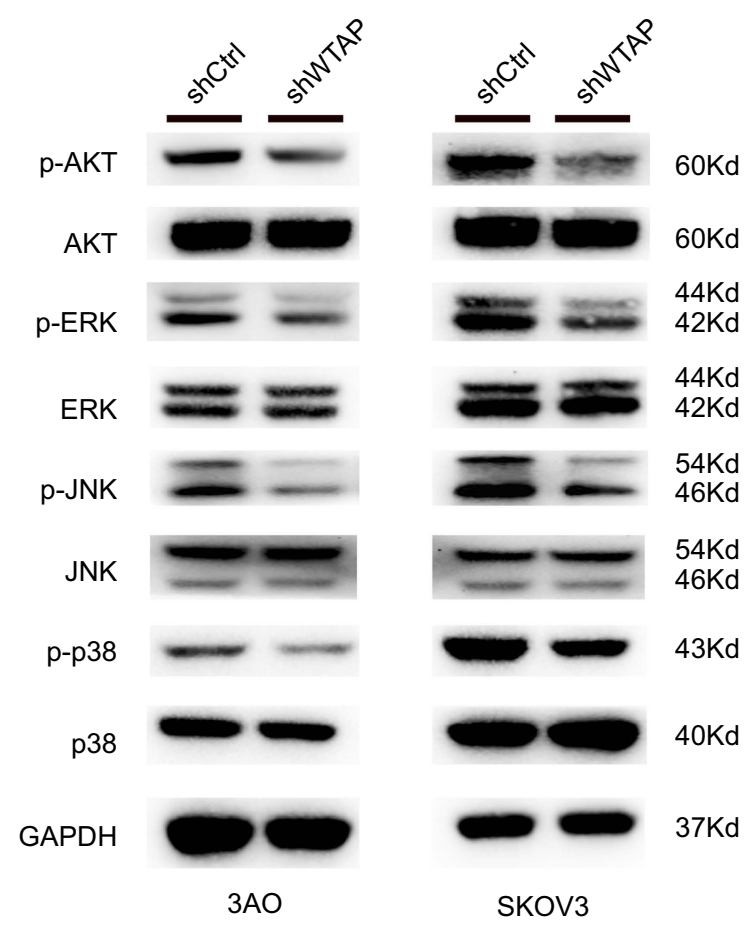

Figure 5 Signaling pathways related to the change of WTAP expression. The effect of WTAP knockdown on the activation of AKT, ERK, JNK, and p38 in $3 A O$ and SKOV3 cell lines.

Besides, its growth stimulating role has been elucidated in several cancers, including pancreatic ductal adenocarcinoma, renal cell carcinoma, and diffuse large B-cell lymphoma. ${ }^{10-12}$ However, the evidence of influence caused by WTAP on ovarian cancer is insufficient.

Using the online tool GEPIA, we serendipitously found that WTAP ranked the third among the 100 most differential survival genes in HGSOC patients, and that high WTAP expression brought patients a worse prognosis and shorter survival time. Further experiments showed that WTAP was expressed higher in HGSOC tissues, indicating WTAP may participate in HGSOC progression or chemo-resistance. Then, we explored the association between WTAP expression and basic clinical information of HGSOC patients. The results demonstrated that high WTAP expression in HGSOC was related to lymph node metastasis.

Apoptosis is regarded as a suicide program when cells experience apoptotic stress, and is ultimately achieved by the activation of caspases and the cleavage of specific proteins. In ovarian cancer, cellular apoptosis is always considered a beneficial process in both preventing and treating. ${ }^{20}$ Apoptosis includes two ways, the extrinsic pathway depends on the recognition of extracellular ligands and surface receptors, while the intrinsic pathway relies on the balance of pro- apoptotic and anti-apoptotic proteins. ${ }^{21}$ The ratio of Bax/Bcl-2 is always considered as an index of the intrinsic apoptosis pathway. However, we found that WTAP knockdown in cell lines did not change the Bax/Bcl-2 level but significantly elevated the ratio of cleaved caspase-3/caspase-3. Hence, WTAP did not affect the apoptosis of 3AO and SKOV3 cell lines by the intrinsic pathway, and the mechanism needs further investigation.

Direct spreading, abdominal cavity implantation, lymphatic metastasis were three main metastasis pathways of ovarian malignant tumors. ${ }^{22}$ These metastatic process is highly associated with the epithelial-mesenchymal transition, which is always defined by the loss of epithelial marker E-cadherin and the gain of mesenchymal marker vimentin, ${ }^{23}$ WTAP could regulate migration and invasion in other cancer cells, ${ }^{10,24}$ but its function in ovarian cancer cells was never reported previously. In this study, we found the down-regulation of WTAP could decrease the migration ability of SKOV3 cell line and change the EMT markers expression correspondingly. This result may explain the phenomenon that we mentioned earlier that WTAP expression was associated with lymph node metastasis in HGSOC patients, indicating that WTAP played a decisive role in the migration ability through the EMT process.

As the combiner of WTAP, WT1 was usually used for diagnostic purpose in gynecological pathology, acting as a biomarker for HGSOC. $^{25}$ Besides, WT1 was also a favorable prognostic factor in HGSOC. ${ }^{26}$ In colorectal cancer, the level of WTAP-WT1 complex decreased when WTAP suffered from degradation, causing free WT1 released, ${ }^{9}$ causing tumor suppression. Thus, we speculated that the remained free WT1 after combining with WTAP would inhibit tumorigenesis, and the inhibition would be abolished if the functional free WT1 is exhausted. Therefore, WTAP and WT1 mRNA of HGSOC samples $(n=563)$ was investigated through the Oncomine Platform (http://www.oncomine.org), revealing that patients with low-WT1 and high-WTAP expression were subjected to the worst survival compared to other genotypes (data not shown). These data were in line with our present results, but further experiments and more insights into details would be required.

To discover the underlying mechanism of WTAP function in $3 \mathrm{AO}$ and SKOV3 cell lines, we checked some widely accepted pathways involved in ovarian cancer progression, such as AKT and MAPK pathways, which were reported in human and mice. ${ }^{27,28}$ In ovarian cancer tissues, the activation of AKT and MAPK pathways played 
essential roles in the process of autophagy, apoptosis, invasion, and chemo-sensitivity. ${ }^{29-34}$ In this study, the phosphorylation levels of AKT, ERK, JNK, and p38 were all decreased due to the down-regulation of WTAP in $3 \mathrm{AO}$ and SKOV3 cell lines, indicating WTAP might affect the activation of AKT and MAPK signaling pathway.

\section{Conclusion}

Taken these data into consideration, we can conclude that WTAP was highly expressed in HGSOC, and was related to the outcome and survival rate of patients. WTAP performed its function by promoting proliferation, migration and inhibited apoptosis in ovarian cancer cell lines. This study provides us a direction that WTAP could be a potential biomarker for the diagnosis of HGSOC patients, but further validation work is required.

\section{Acknowledgments}

This work is supported by grants from the Medical and Health Technology Program Foundation of Zhejiang Province (no. 2017186075).

\section{Disclosure}

The authors report no conflicts of interest in this work.

\section{References}

1. LA T, Trabert B, DeSantis CE, et al. Ovarian cancer statistics, 2018. CA Cancer J Clin. 2018;68:284-296. doi:10.3322/caac.21456

2. Della PC, Tonini G, Santini D, et al. Low Grade Serous Ovarian Carcinoma: from the molecular characterization to the best therapeutic strategy. Cancer Treat Rev. 2015;41:136-143. doi:10.1016/j. ctrv.2014.12.003

3. Konecny GE, Wang C, Hamidi H, et al. Prognostic and therapeutic relevance of molecular subtypes in high-grade serous ovarian cancer. J Natl Cancer Inst. 2014;106:dju249.

4. Lheureux S, Braunstein M, Oza AM, Braunstein M, Oza AM. Epithelial ovarian cancer: evolution of management in the era of precision medicine. Cancer J Clin J Clin. 2019. doi:10.3322/caac.21559

5. Little NA, Hastie ND, Davies RC, Hastie ND, Davies RC. Identification of WTAP, a novel Wilms' tumour 1-associating protein. Hum Mol Genet. 2000;9:2231-2239. doi:10.1093/oxfordjournals.hmg.a018914

6. Yang L, Han Y, Suarez Saiz F, Saurez Saiz F, Minden MD. A tumor suppressor and oncogene: the WT1 story. Leukemia. 2007;21:868-876. doi:10.1038/sj.leu.2404624

7. Lu J, Gu Y, Li Q, et al. Wilms' tumor 1 (WT1) as a prognosis factor in gynecological cancers: a meta-analysis. Medicine (Baltimore). 2018;97:e11485. doi:10.1097/MD.0000000000011485

8. Carter JH, Deddens JA, Mueller G, et al. Transcription factors WT1 and p53 combined: a prognostic biomarker in ovarian cancer. $\mathrm{Br}$ J Cancer. 2018;119:462-470. doi:10.1038/s41416-018-0191-x

9. Zhang J, Tsoi H, Li X, et al. Carbonic anhydrase IV inhibits colon cancer development by inhibiting the Wnt signalling pathway through targeting the WTAP-WT1-TBL1 axis. Gut. 2016;65:1482-1493. doi:10.1136/gutjnl-2014-308614
10. Li BQ, Huang S, Shao QQ, et al. WT1-associated protein is a novel prognostic factor in pancreatic ductal adenocarcinoma. Oncol Lett. 2017;13:2531-2538. doi:10.3892/ol.2017.5784

11. Tang J, Wang F, Cheng G, et al. Wilms' tumor 1-associating protein promotes renal cell carcinoma proliferation by regulating CDK2 mRNA stability. J Exp Clin Cancer Res. 2018;37:40. doi:10.1186/ s13046-018-0706-6

12. Kuai Y, Gong X, Ding L, et al. Wilms' tumor 1-associating protein plays an aggressive role in diffuse large B-cell lymphoma and forms a complex with BCL6 via Hsp90. Cell Commun Signal. 2018;16:50. doi:10.1186/s12964-018-0258-6

13. Van Nieuwenhuysen E, Busschaert P, Neven P, et al. The genetic landscape of 87 ovarian germ cell tumors. Gynecol Oncol. 2018;151:61-68. doi:10.1016/j.ygyno.2018.08.013

14. Tang Z, Li C, Kang B, Gao G, Li C, Zhang Z. GEPIA: a web server for cancer and normal gene expression profiling and interactive analyses. Nucleic Acids Res. 2017;45:W98-W102. doi:10.1093/nar/ gkx247

15. Cancer Genome Atlas Research Network. Integrated genomic analyses of ovarian carcinoma. Nature. 2011;474:609-615. doi:10.1038/ nature 10166

16. Jayson GC, Kohn EC, Kitchener HC, Ledermann JA. Ovarian cancer. Lancet. 2014;384:1376-1388. doi:10.1016/S0140-6736(13)62146-7

17. He L, Li J, Wang X, et al. The dual role of N6-methyladenosine modification of RNAs is involved in human cancers. J Cell Mol Med. 2018;22:4630-4639. doi:10.1111/jcmm.13804

18. Schwartz S, Mumbach MR, Jovanovic M, et al. Perturbation of m6A writers reveals two distinct classes of mRNA methylation at internal and 5' 5' sites. Cell Rep. 2014;8:284-296. doi:10.1016/j. celrep.2014.05.048

19. Lesbirel S, Viphakone N, Parker M, et al. The m(6)A-methylase complex recruits TREX and regulates mRNA export. Sci Rep. 2018;8:13827. doi:10.1038/s41598-018-32310-8

20. Ichim G, Tait S. A fate worse than death: apoptosis as an oncogenic process. Nat Rev Cancer. 2016;16:539-548. doi:10.1038/ nrc. 2016.58

21. Fernald K, Kurokawa M. Evading apoptosis in cancer. Trends Cell Biol. 2013;23:620-633. doi:10.1016/j.tcb.2013.07.006

22. Pastushenko I, Blanpain C. EMT transition states during tumor progression and metastasis. Trends Cell Biol. 2019;29:212-226. doi:10.1016/j.tcb.2018.12.001

23. Klymenko Y, Kim O, Stack M. Complex determinants of epithelial: mesenchymal phenotypic plasticity in ovarian cancer. Cancers (Basel). 2017;9:104.

24. Jo H, Shim H-E, Han M-E, et al. WTAP regulates migration and invasion of cholangiocarcinoma cells. $J$ Gastroenterol. 2013;48:1271-1282. doi:10.1007/s00535-013-0748-7

25. Hylander B, Repasky E, Shrikant P, et al. Expression of Wilms tumor gene (WT1) in epithelial ovarian cancer. Gynecol Oncol. 2006;101:2-7. doi:10.1016/j.ygyno.2005.09.052

26. Taube ET, Denkert C, Sehouli J, et al. Wilms tumor protein 1 (WT1) - not only a diagnostic but also a prognostic marker in highgrade serous ovarian carcinoma. Gynecol Oncol. 2016;140:494-502. doi:10.1016/j.ygyno.2015.12.018

27. Wagner EF, Nebreda AR. Signal integration by JNK and p38 MAPK pathways in cancer development. Nat Rev Cancer. 2009;9:537-549. doi: $10.1038 / \mathrm{nrc} 2694$

28. Xu Y, Na L, Xiang R, Sun P. Emerging roles of the p38 MAPK and $\mathrm{PI} 3 \mathrm{~K} / \mathrm{AKT} / \mathrm{mTOR}$ pathways in oncogene-induced senescence. Trends Biochem Sci. 2014;39:268-276. doi:10.1016/j.tibs.2014. 04.004

29. Lim V, Zhu H, Diao S, Hu L, Hu J. PKP3 interactions with MAPK-JNK-ERK1/2-mTOR pathway regulates autophagy and invasion in ovarian cancer. Biochem Biophys Res Commun. 2019;508:646-653. doi:10.1016/j.bbrc.2018.11.163 
30. Nie X, Liu C, Guo Q, et al. TMEFF1 overexpression and its mechanism for tumor promotion in ovarian cancer. Cancer Manag Res. 2019;11:839-855. doi:10.2147/CMAR.S186080

31. Wu J, Sun Y, Zhang P-Y, et al. The Fra-1-miR-134-SDS22 feedback loop amplifies ERK/JNK signaling and reduces chemosensitivity in ovarian cancer cells. Cell Death Dis. 2016;7:e2384. doi:10.1038/cddis.2016.289

32. Ghasemi A, Hashemy SI, Aghaei M, Panjehpour M. Leptin induces matrix metalloproteinase 7 expression to promote ovarian cancer cell invasion by activating ERK and JNK pathways. $J$ Cell Biochem. 2018;119:2333-2344. doi:10.1002/jcb.26396
33. Yang H, Mao W, Rodriguez-Aguayo C, et al. Paclitaxel sensitivity of ovarian cancer can be enhanced by knocking down pairs of kinases that regulate MAP4 phosphorylation and microtubule stability. Clin Cancer Res. 2018;24:5072-5084. doi:10.1158/1078-0432.CCR-180504

34. Lu L, Wang J, Wu Y, Wan P, Yang G. Rap1A promotes ovarian cancer metastasis via activation of ERK/p38 and notch signaling. Cancer Med. 2016;5:3544-3554. doi:10.1002/ cam4.946

\section{Publish your work in this journal}

OncoTargets and Therapy is an international, peer-reviewed, open access journal focusing on the pathological basis of all cancers, potential targets for therapy and treatment protocols employed to improve the management of cancer patients. The journal also focuses on the impact of management programs and new therapeutic agents and protocols on patient perspectives such as quality of life, adherence and satisfaction. The manuscript management system is completely online and includes a very quick and fair peer-review system, which is all easy to use. Visit http://www.dovepress.com/ testimonials.php to read real quotes from published authors. 